\title{
Detection of Symmetry and Repetition in One and Two Objects Structures Versus Strategies
}

\author{
Arno Koning and Johan Wagemans
}

University of Leuven, Belgium

\begin{abstract}
Symmetry is usually easier to detect within a single object than in two objects (one-object advantage), while the reverse is true for repetition (two-objects advantage). This interaction between regularity and number of objects could reflect an intrinsic property of encoding spatial relations within and across objects or it could reflect a matching strategy. To test this, regularities between two contours (belonging to a single object or two objects) had to be detected in two experiments. Projected three-dimensional (3-D) objects rotated in depth were used to disambiguate figure-ground segmentation and to make matching based on simple translations of the two-dimensional (2-D) contours unlikely. Experiment 1 showed the expected interaction between regularity and number of objects. Experiment 2 used two-objects displays only and prevented a matching strategy by also switching the positions of the two objects. Nevertheless, symmetry was never detected more easily than repetition in these two-objects displays. We conclude that structural coding, not matching strategies, underlies the one-object advantage for symmetry and the two-objects advantage for repetition.
\end{abstract}

Keywords: visual regularity, symmetry, repetition, 3-D object perception, perceptual grouping, figure-ground organization, structural coding

In visual perception research, the inverse problem refers to the task of the visual system to reconstruct a representation of the three-dimensional (3-D) outside world based mostly on two-dimensional (2-D) information. Fortunately, there are many visual cues in the environment that help us structure the incoming information into an organized whole. Regularities like symmetry and repetition are examples of such visual cues. Many objects, both natural and man-made, show some form of regularity, be it bilateral mirror symmetry, rotational symmetry, or repetition (e.g., Tyler, 1995). In fact, regularities like these are closely related in their mathematical basis (Palmer, 1983; Van der Helm \& Leeuwenberg, 1991; Wagemans, 1995; Wagemans, Van Gool, Swinnen, \& Van Horebeek, 1993), even to the extent that in the mathematical sense all these regularities are symmetries. The way to distinguish these regularities is by referring to the transformation that is used to create them (i.e., reflection, rotation, translation, respectively). For example, one can create bilateral mirror symmetry ("symmetry" from now on) and repetition starting from the contour presented in Figure 1a. One way to create a repetition with this jagged contour is to copy it and then translate it (see Figure 1b). Analogously, one can create symmetry with the same contour in Figure 1a by using an additional transformation, a reflection (see Figure 1c). Using this way to create symmetry and repetition, Mach (1885/1959) noted the following paradox: Even though repetition can be accomplished by a single translation, while symmetry requires both a translation and a reflection, symmetry seems easier to detect than repetition.

To investigate Mach's paradox further, Baylis and Driver (1994) performed one experiment in which participants had to judge whether objects had symmetric (see Figure 1d and 1e) or random contours, and another one in which participants had to judge whether objects had repeated (see Figure if and $1 \mathrm{~g}$ ) or random contours. In both experiments, the number of discontinuities in the contours of the objects was varied. It was found that for symmetric contours reaction times and error rates were not affected when the number of discontinuities increased (cf. Figure 1d with 1e). For repeated contours, however, an increasing number of discontinuities in the contours (cf. Figure $1 \mathrm{f}$ with $1 \mathrm{~g}$ ) resulted in increasing reaction times and error rates. Baylis and Driver explained this symmetry advantage by pointing out that the polarities of convexities and concavities are the same in case of symmetric contours but reversed for repeated contours. As a result, symmetric objects have corresponding part decompositions (Hoffman \& Richards, 1984) on both sides of the axis of symmetry, but objects showing repeated contours do not have such corresponding parts. In other words, for symmetric objects, convex points and concave points are the same on both sides of the symmetry axis, whereas for objects with repeated contours what is convex on one side of the object is concave on the other side of the object and vice versa.

This explanation cannot be complete, however, because both Baylis and Driver (1995) and Bertamini, Friedenberg, and Kubovy (1997) obtained differential effects for symmetry and repetition when contours belong to one object as opposed to two objects. More specifically, Bertamini et al. found that symmetry judgments were easier when the contours appeared to belong to a single object compared to when they appeared to belong to two objects (i.e., a one-object advantage, cf. Figure $1 \mathrm{~h}$ to $1 \mathrm{i}$ ), whereas repetition judgments showed a 


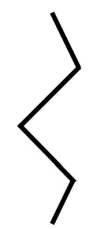

(a)

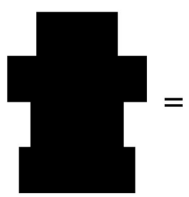

(d)

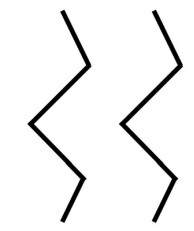

(b)

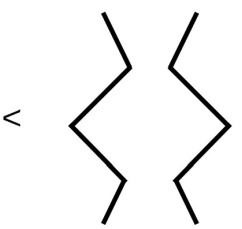

(c)

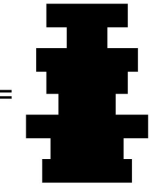

(e)



(f)

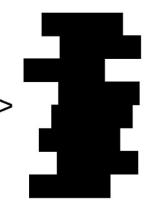

(g)

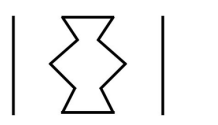

(h)

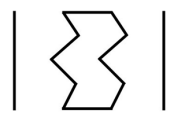

(j)

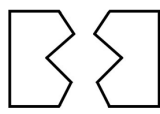

(i)
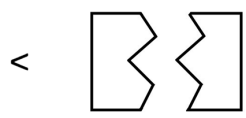

(k)

Figure 1. (a) A single jagged contour. (b) By copying and translating the contour in (a), a repetition is constructed. (c) By copying, translating, and reflecting the contour in (a), a symmetry is constructed. (d-g) After Baylis and Driver (1994). (d) A bilaterally symmetric object with relatively few discontinuities in its contour. (e) A bilaterally symmetric object with relatively many discontinuities in its contour. (f) An object with relatively few discontinuities in its repeated contour. (g) An object with relatively many discontinuities in its repeated contour. (h-k) After Bertamini, Friedenberg, and Kubovy (1997). (h) A one-object display showing a symmetric object. (i) A two-objects display showing a symmetry. (j) A one-object display showing an object with repeated contours. (k) A two-objects display showing a repetition between the jagged contours. Note that the small symbols $(<,>,=)$ refer to the detectability of regularity, where " $>$ " indicates "easier to detect than" and " $<$ " indicates "harder to detect than".

reversed pattern of results (i.e., a two-objects advantage or a one-object cost, cf. Figure $1 \mathrm{j}$ to $1 \mathrm{k}$ ). This interaction between type of regularity and number of objects cannot be explained on the basis of similarities or differences in part decomposition. That is to say, in the two-objects situations the two symmetry halves also have corresponding parts (see Figure 1i), whereas the two repetition halves do not have corresponding parts (see Figure 1k). Bertamini et al. argued that in the twoobjects situation with a repetition between the contours, the two separate pieces could be mentally translated in the image plane, resulting in a fit between the two pieces in the same way a key fits a lock, thereby producing an advantage for repeated contours. Similar results were also found by Baylis and Driver (1995), who suggested that this repetition advantage for the two-objects situation could be due to a mental strategy in

which the objects with repeated contours are treated as pieces of a jigsaw puzzle.

At this point, two important clarifications are needed. First, the research described above has focused on the detection of the regularity between two contours and they considered the effect of the object's context by connecting the pairs of contours in different ways. However, the resulting objects are themselves not always symmetric or repeated (see Figure 1). Although most configurations with reflected pairs of contours give rise to symmetric objects (except Figure 1i), the situation is particularly problematic for repeated pairs of contours. As pointed out by Strother and Kubovy (2003), the mathematical condition of invariance under translation only holds for infinite patterns (see also Kubovy \& Wagemans, 1995; Kubovy, Holcombe, \& Wagemans, 1998; Wagemans, 1995; Wagemans et al., 1993). Moreover, as pointed out by Csatho, Van der Vloed, and Van der Helm (2003), constructing a set of repeated contours such that the polarities of convexities and concavities differ, as is the case in all "repetition" displays in Figure 1 except for Figure 1b, does not result in a repetition at all, but rather in an antirepetition. This problem does not arise when dot patterns are used. For example, Corballis and Roldan (1974) have tested regularity detection in dot patterns with symmetry or repetition and they manipulated "objectness" by presenting the two dot patterns used to create the regularity either close together (one object) or further apart (two objects). Based on their results, Corballis and Roldan also suggested that symmetry is more salient than repetition when participants are asked to perceive stimuli "holistically" (i.e., as one object), a conclusion that seems very similar to those of Baylis and Driver (1995) and Bertamini et al. (1997). Because in our study we will also work with contours that are connected in certain ways and because we are also interested in the effect of the object context, we will retain the terminology of symmetry and repetition (for reasons of consistency with the literature we are building on), although antirepetition would be a mathematically more appropriate terminology.

A second clarification concerns the specification of the interaction between type of regularity and number of objects. Originally, it has been formulated as a shift from a symmetry advantage for one-object displays (i.e., Mach's paradox in one-object displays) to a repetition advantage for two-objects displays (i.e., disappearance of Mach's paradox in two-objects displays). However, an interaction also occurs when one-object displays yield better performance than twoobjects displays when symmetry must be detected (i.e., oneobject advantage for symmetry) and the reverse occurs when repetition must be detected (i.e., one-object cost or twoobjects advantage for repetition). In principle, these two types of changes between pairwise comparisons from one context to another can occur more or less independently from one another but both would give rise to a statistically significant two-way interaction between type of regularity and number of objects. In our study, we are more interested in the second way in which the interaction will reveal itself than in the first way for reasons that will become clear shortly. 
Let us return now to the specific explanations of Baylis and Driver (1994) and Bertamini et al. (1997) for their results. The one-object advantage for symmetry over twoobjects situations is explained in terms of structural differences (Baylis \& Driver, 1994). In contrast, the two-objects advantage for repetition over one-object situations is explained in terms of a specific strategy of the observer (Baylis \& Driver, 1995; Bertamini et al., 1997). In this study, however, we want to argue that it seems preferable, for reasons of simplicity, to explain the interaction between type of regularity and number of objects in terms of either one of these effects alone rather than both of them in combination. Based on the current literature, it is very difficult to distinguish the two accounts. That is, in the above-mentioned studies, the perceived number of objects was manipulated by altering the belongingness of the contours of interest to different parts in the image, using grouping principles such as connectedness and closure. Both Baylis and Driver (2001) and Bertamini et al. (1997) mentioned that such manipulations were probably not always effective. For example, in the two-objects situation in which the contours are symmetric (Figure 1i), it is also possible to perceive the central area as figure and then we are back in the one-object case. This ambiguity with respect to figure-ground segmentation of these two-objects situations is also highly reminiscent of the famous faces/vase illusion by Rubin (1921). Indeed, when Baylis and Driver (2001) manipulated the surrounding (background) region of two-objects situations in such a way that now the central part of the stimulus was more likely to be perceived as (a symmetrical) figure, performance approached that of one-object situations.

Nevertheless, if the closed regions in Figures $1 \mathrm{i}$ and $1 \mathrm{k}$ are actually represented as objects that are segregated from the background, as intended by Bertamini et al. (1997), then the two-objects advantage that these authors found for repetition could be explained in two ways. First, the one-object advantage for symmetry and the two-objects advantage for repetition could reflect an intrinsic property of the way in which the visual system processes and represents visual regularities of objects. Despite considerable differences between them (e.g., Van der Helm \& Leeuwenberg, 1996, 1999; Wagemans, 1999), both process models (e.g., Jenkins, 1983; Van der Helm \& Leeuwenberg, 1999; Wagemans, 1995, 1997, 1999; Wagemans, Van Gool, \& d'Ydewalle, 1991; Wagemans et al., 1993) and representational models (e.g., Van der Helm \& Leeuwenberg, 1996) are compatible with the notion that symmetry is a strong one-object cue, while repetition is a strong two-objects cue. This structural difference is especially salient in the holographic approach by Van der Helm and Leeuwenberg $(1996,1999)$ but it also follows from the bootstrap model (Wagemans, 1995, 1997; Wagemans et al., 1993), within which the pairwise matches between symmetric elements establish a strong overall structural network connected by the symmetry axis, whereas the pairwise matches between repeated elements are not structurally connected by an overall axis. In other words, symmetry, being a one-object cue, may be detected better in one-object cases than in two-objects cases, whereas for repetition, being a two-objects cue, the reverse is more likely, even when lock-and-key matching is made unlikely. Second, the two-objects advantage for repetition could be due to a matching strategy by the participant (i.e., lock-and-key matching in terms of Bertamini et al.). In other words, the two-objects advantage would then rather be an exception caused by the special situation of the two objects fitting perfectly well together when closing the gap between them.

To address the issue whether the interaction between type of regularity and number of objects is better explained by structural differences or specific matching strategies, we performed two experiments in which the participant had to judge whether a particular regularity (symmetry or repetition) was present or not. In Experiment 1, we start from the previously reported one-object symmetry advantage and two-objects repetition advantage, but we use projections of 3-D objects instead of 2-D objects. Moreover, the 3-D objects will be presented at an angle away from the line of sight of the observer. This allows for a more direct investigation of visual regularity detection as a function of type of regularity (symmetry vs. repetition) and number of objects (one vs. two). That is, if the intrinsic coding of the object(s) is important, using slanted 3-D objects will lead to the strongest possible interaction effect because of two reasons. First, the figure-ground ambiguity in the two-objects displays will be reduced, removing some one-object effects from the corresponding results. Second, the objectness of the stimuli will be enhanced, preventing a simple planar translation between the 2-D contours. In Experiment 2, we will more closely investigate spatial positioning of the objects in the twoobjects cases to exclude a lock-and-key or jigsaw matching strategy in half of the conditions. Additionally, the grouping strength of the two objects will be varied.

\section{Experiment 1}

This experiment wants to make a stronger case for the role of object structure in determining visual regularity detection than the available literature so far by introducing two novel characteristics into the stimulus displays. First, the use of projected 3-D objects in this study is likely to result in a more clear segmentation of the figures from the background than that of the objects in the studies by both Baylis and Driver (1995) and Bertamini et al. (1997), where the interaction between type of regularity and number of objects may have been suppressed by unintended figural interpretations of the background area between the two objects. A second strength of this experiment is the theoretical advantage of being able to attribute effects to object-based regularity rather than image-based regularity. That is, by turning away the frontal sides of the 3-D objects from the line of sight, the contours are no longer literal copies of one another in the image plane, thereby reducing the degree of image-based regularity. If object-based regularity underlies the previously obtained interaction between type of regularity and number of objects, it should be preserved in this experiment and become even stronger perhaps for the reasons given above. 


\section{Methods}

\section{Participants}

Twenty graduate and undergraduate students of psychology at the University of Leuven (19-27 years) performed the experiment. The undergraduate students were given course credit or were paid for their time. The graduate students participated voluntarily. All participants reported normal or corrected-to-normal vision and were naïve regarding the goals and details of the experiment.

\section{Stimuli}

Stimulus construction started in 3DToolkit version 2.5 (Electric Image, Inc.). Sixteen different jagged contours were created by systematically varying the extents of three arbitrarily created protrusions (see Figure 2). For the oneobject situations with repetition, each contour (A through P) was copied and translated, after which the two contours were connected using straight lines. The resulting shape was then extruded to create a 3-D object (e.g., the object in panel 3 of Figure 3). For the one-object situations with symmetry, the same procedure was followed except that the copied contour was also reflected (e.g., the object in panel 1 of Figure 3). The two-objects situations were created similarly, except that each contour (A through P) was now not connected to its copied counterpart, but to straight lines drawn

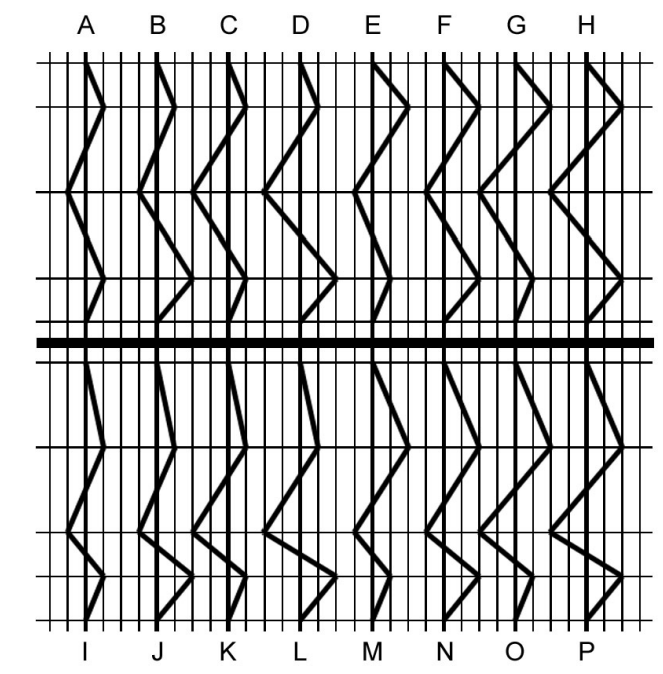

Figure 2. The 16 contours used to create the 3-D objects in Experiment 1. For the first set of contours $(\mathrm{A}-\mathrm{H})$, three deviations from straight lines occurred at fixed vertical positions and their sizes were varied systematically to create eight different contours. For the second set (I-P), deviations from straight lines occurred at three different vertical positions along the contours, but as with the first set, the sizes of the protrusions were varied systematically to create eight different contours. on the contour's opposite side, after which the shapes were extruded to create 3-D objects (see Figure 3, panels 2 and 4). In addition, two rectangular blocks were created and placed on both sides of the one-object situations. This way, the horizontal (as well as the vertical) extents of all stimuli were the same (as in Bertamini et al., 1997; see also Figure $1 \mathrm{~h}$ and $1 \mathrm{j}$ ). To balance the directions of the protrusions of all objects, the contours were also mirrored leftright. For example, tracing the central object in the upper left cell of Figure 3 from top to bottom, the contours of the object first diverge, then converge, then diverge again, and finally converge again. Left-right mirroring of the contours in this case resulted in an object that had the same protrusions, except now the sequence of divergence and convergence of the contours was reversed. All objects were placed in two different orientations in depth. That is, the objects were placed once at an angle of $45^{\circ}$ (with respect to the frontal plane) to the right and once at an angle of $45^{\circ}$ to the left.

To create the objects without regularity, different contours were paired. For example, contour A was paired with contour P (see Figure 2), contour B was paired with contour $\mathrm{O}$, and so on for the remaining contours. In total, 512 objects were rendered: Sixteen contours $\times 2$ regularities (symmetry and repetition) $\times 2$ numbers of objects (oneobject and two-objects situations) $\times 2$ orientations of the objects $\left(45^{\circ}\right.$ to the left and $45^{\circ}$ to the right with respect to the frontal plane) $\times 2$ by left-right mirroring of the contours $\times 2$ for presence/absence of regularity.

\section{Procedure}

Trials were presented on a 17 -in. monitor at a resolution of $1024 \times 768$ pixels controlled by a Pentium-III computer, running under Windows 98. The participants were seated at about $90 \mathrm{~cm}$ from the monitor. Each stimulus covered an area of $8.5 \times 10.5 \mathrm{~cm}$ on the screen (i.e., a visual angle of about $5^{\circ}$ horizontally by $7^{\circ}$ vertically). E-prime (PST Inc.) was used to run the experiment. Participants sat in a completely darkened room while performing the experiment, with the only light reflecting from the monitor. The experiment was divided into two blocks: One block in which the contours were symmetric or not and one block in which the contours were repeated or not. The order of the blocks (symmetry first or repetition first) was counterbalanced across participants. Each block was preceded by 20 randomly selected practice trials. Each trial started with a fixation cross $(750 \mathrm{~ms})$, followed by a stimulus display, which remained on the screen until the participant responded. It was the participant's task to judge as quickly as possible whether the jagged contours in the stimulus showed the required regularity or not. Using an AZERTY keyboard, half of the participants had to press the "w" when the regularity was present, and press the "," when it was absent. The other half received the opposite key assignment. During the practice trials, the participant's response was followed by visual feedback indicating whether it was correct or incorrect. In the experimental trials, no feedback was given, but instead a blank screen $(750 \mathrm{~ms})$ appeared, which was followed by 


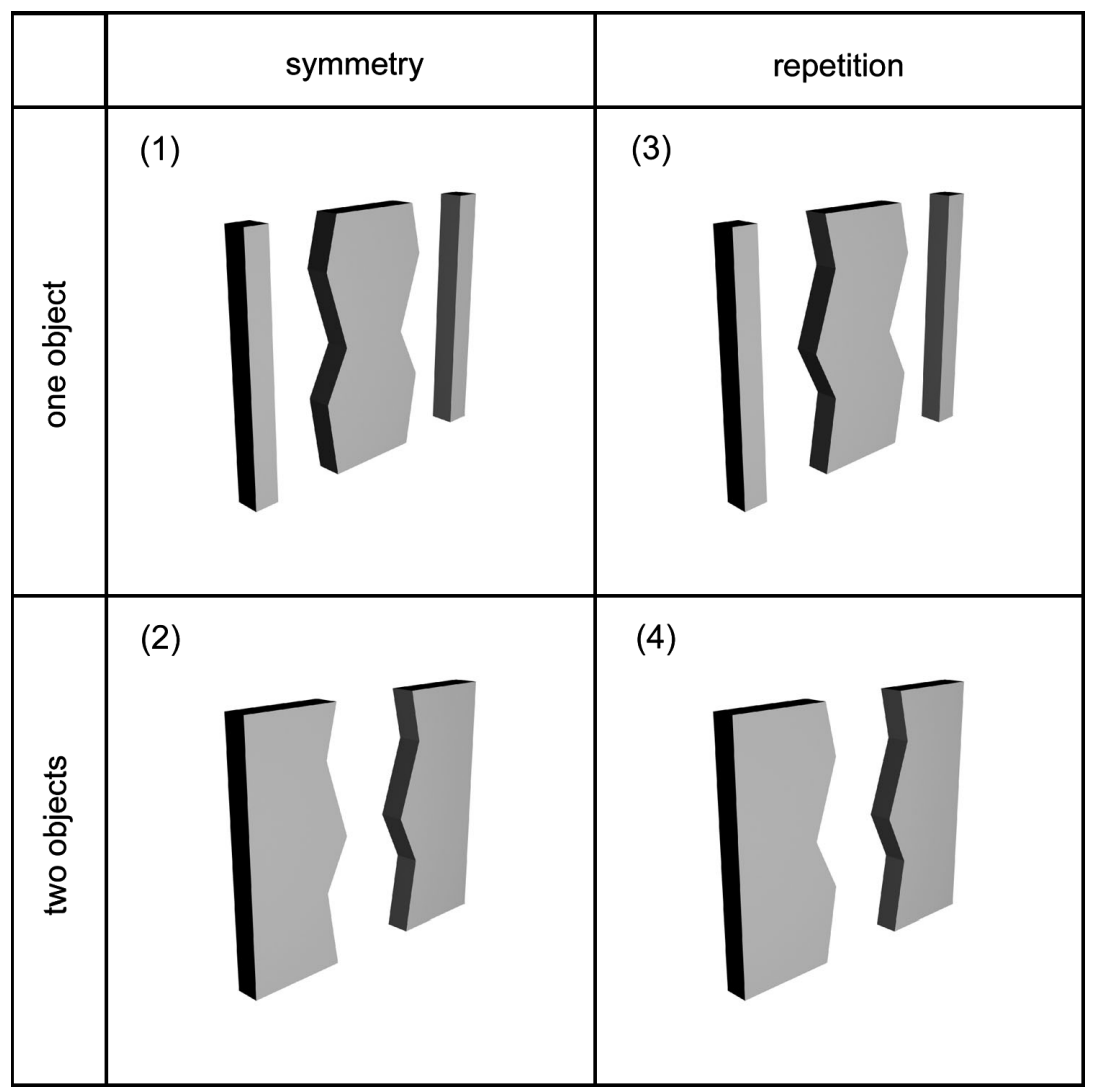

Figure 3. Examples of the 3-D objects used in Experiment 1. The top row shows the one-object displays for both the symmetric contours as well as the repeated contours. The bottom row shows the two-objects displays for both the symmetric contours as well as the repeated contours. Note that vertical bars are added in the one-object displays to equate the horizontal extent to that of the two-objects displays.

the fixation cross indicating that the next trial started. All 512 trials were presented in a single session. The entire experiment took about 30 min to complete. Reaction times (RTs, to the nearest $\mathrm{ms}$ ) and error rates were measured.

\section{Results and Discussion}

There was an overall error rate across participants for all trials of $7.5 \%$. Regularities (either symmetries or repetitions) were missed in $6.1 \%$ of the trials and $9.0 \%$ of the trials containing no regularities were incorrectly reported to contain a regularity. Trials that did not contain a regularity were not analyzed further. There was no speed-accuracy trade-off. Preliminary analyses revealed no main or interaction effects with respect to the variable "orientation", therefore the data were pooled across this variable. For correct trials in which the regularity was present, mean RT was analyzed using a two-way repeated-measures ANOVA with variables regularity (2) and number of objects (2). A main effect was found for regularity, $F(1,19)=29.89, p<.001, \eta_{\mathrm{P}}{ }^{2}=0.611$. Symmetric contours $($ Mean $=922 \mathrm{~ms}, S E M=60 \mathrm{~ms})$ were responded to faster than repeated contours (Mean $=1217 \mathrm{~ms}$, $S E M=99 \mathrm{~ms})$. More importantly, a strong interaction effect was found between regularity and number of objects, $F(1,19)=22.41, p<.001, \eta_{\mathrm{P}}{ }^{2}=0.541$ (see Figure 4). Contrast comparison revealed that symmetric contours were responded to faster when they belonged to one object than when they belonged to two objects, $F(1,19)=16.99$, $p<.005$, a significant one-object advantage. In contrast, repeated contours were responded to slower when they belonged to one object than when they belonged to two objects, $F(1,19)=15.39, p<.005$, a significant one-object cost.

The RT analysis revealed a main effect of regularity with an advantage for symmetric contours over repeated contours. This is in line with the findings by Baylis and Driver (1994, 1995, 2001) and Bertamini et al. (1997). More importantly, the interaction between type of regularity and number of objects was also quite strong. Thus, using projected 3-D objects, symmetric contours were identified more quickly when they belonged to one object than when they belonged to two objects. In contrast, repeated contours were identified more quickly when they belonged to two objects than when they belonged to one object. Moreover, by presenting 3-D objects with frontal surfaces turned away from the line of sight, we were able to provide evidence that the previously reported interaction between type of regularity 


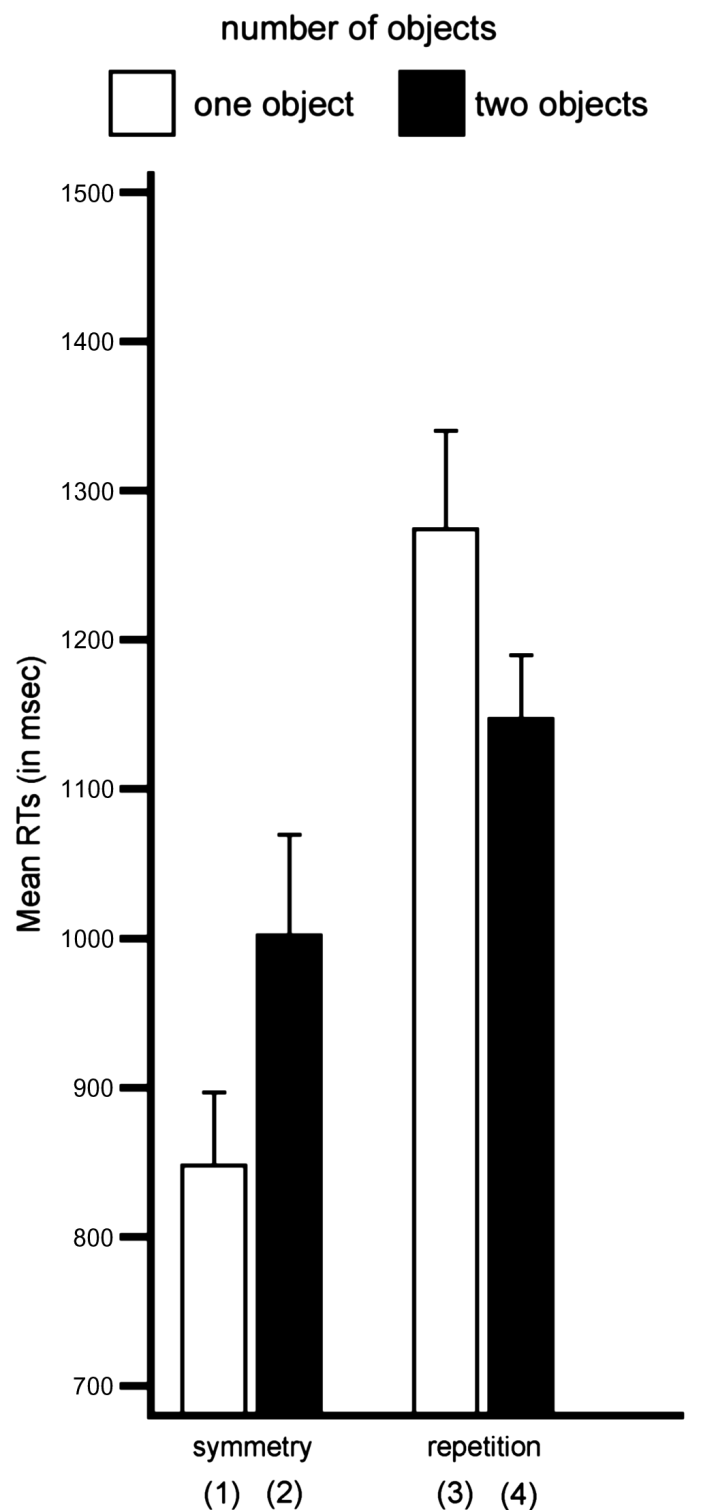

Figure 4. Results of Experiment 1. Graph of the mean reaction times (RTs) as a function of regularity (symmetry and repetition) and number of objects (one and two). Error bars represent one standard error of the mean (representing the variability of each data point across participants). The numbers in parentheses on the $X$ axis refer to the panels in Figure 3.

and number of objects depends primarily on object structure rather than on a matching strategy that could be used by the observers.

The present results could, however, be interpreted differently. That is, the 3-D objects rotated in depth that are used here only prevent a simple one-to-one (image-based) matching strategy. It is still possible that participants used a somewhat more complicated mental translation strategy, where they first recovered the slant of the plane connecting the two objects and then applied a matching strategy. Thus, as mentioned before, because of the gaps in the two-objects situations, the results could be due to either matching strategies by the participants or structural encoding differences in the visual system (i.e., symmetry is a one-object cue, whereas repetition is a two-objects cue). To further dissociate matching strategies from structural differences, two-objects situations will be examined more closely in Experiment 2 by comparing two-objects situations similar to those of Experiment 1 with two-objects situations in which the positions of the objects are switched. This way, two objects are still present, but a matching strategy is no longer likely.

\section{Experiment 2}

In this second experiment, we investigated regularity detection using two-objects situations only. The positions of the two objects, and thus also the positions of the jagged contours, relative to each other were varied. The jagged contours of the objects could either face each other (cf., the two-objects situations in the bottom row of Figure 3) or the objects could switch places causing the jagged contours to face away from each other. However, when the objects in the two-objects situations in Figure 3 are switched, the resulting gap between the objects becomes highly regular. That is, the resulting gap has a rectangular shape and, in terms of regularity, could then be described as having both a symmetric and a repeated character. Therefore, in this experiment we also varied the regularity of the gap between the two objects. Thus, there were two main manipulations in this experiment: The jagged contours could be facing or nonfacing, and the strength of perceptual grouping of the objects was varied based on regularity type and gap size.

To accomplish this, two types of contours were defined: (i) contours of interest, jagged contours similar to the ones used in Experiment 1 for which regularity detection has to be performed; and (ii) irrelevant contours, curved contours that are clearly different from the contours of interest (see also Figure 5) and irrelevant to the regularity detection task. Using these contours, 3-D objects were created such that when the contours of interest were facing, the irrelevant contours were nonfacing and vice versa. As a result, in the stimulus displays, either the horizontal visual extent between the contours of interest would be different between the facing and the nonfacing conditions or the size of the gaps between these two conditions would be different. We decided to keep the visual extent between the contours of interest the same throughout the experiment because the matching of the contours of interest is the process of major interest. Of course, the specific relations between the irrelevant contours will also affect the strength of the perceptual grouping of the two-objects situations when the contours of interest are nonfacing (see also Figure 6). Further, like in Experiment 1, the contours of interest of the two objects could be symmetric, repeated, or random, but now also the irrelevant contours of the two objects could be symmetric, repeated, or random.

Although the predictions of the two accounts can no longer be specified in terms of one- versus two-objects 


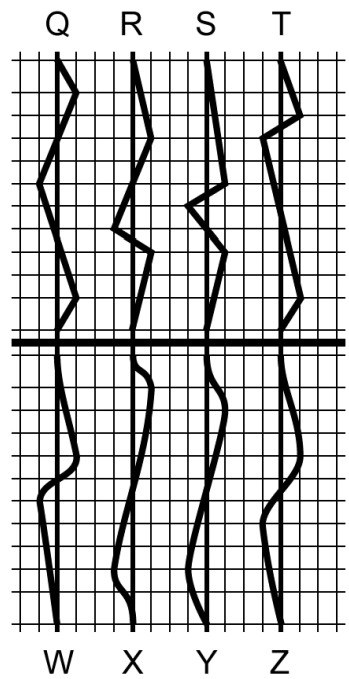

Figure 5. The contours created for Experiment 2. The contours of interest are presented at the top; the irrelevant contours are presented at the bottom. The creation of these contours was similar to that of Experiment 1.

displays in this second experiment, several expectations can still be formulated. First, because two-objects displays are used performance for repetition is not expected to be worse than performance for symmetry according to the structural account. In the case of nonfacing contours, the strategic account predicts more difficulties for repetition and hence symmetry might be easier than repetition in that condition. Second, the irrelevant contours are likely to influence perceptual grouping of the two objects. As a result, regularity detection of the contours of interest is expected to vary depending on the type of irrelevant contour. More specifically, if object structure is important, perceptual grouping of the two objects in the display is more likely to influence symmetry judgments than repetition judgments. For instance, adding a second symmetry between the irrelevant contours may then have a beneficial effect both when the contours of interest are facing and nonfacing because the overall stimulus display is then symmetric (at the object level, not the image level). With repetition being a twoobjects cue, this is less likely to occur for repetition judgments. Alternatively, if the previously obtained two-objects repetition advantage is due to a matching strategy, it may be expected that repetition displays will be more influenced by the regularity of the irrelevant contours (because of possible confusions in the specific contours to be matched).

Finally, it should be noted that the current manipulations of the two types of contours, combined with the nature of the task, may also lead to facilitation or interference effects at the response level. Even though the participants are told to ignore the irrelevant contours and judge the regularity of the

\begin{tabular}{|c|c|c|c|c|}
\hline & \multicolumn{2}{|c|}{ symmetric contours of interest } & \multicolumn{2}{|c|}{ repeated contours of interest } \\
\hline & facing & nonfacing & facing & nonfacing \\
\hline 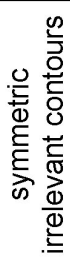 & (1) & (4) & (7) & \\
\hline 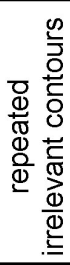 & (2) & (5) & (8) & (11) \\
\hline 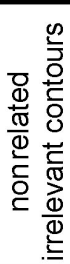 & $\Gamma^{(3)}$ & (6) & 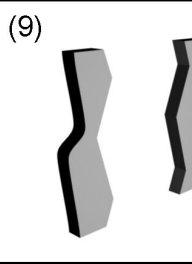 & $\sum^{(12)}$ \\
\hline
\end{tabular}

Figure 6. Examples of the 3-D objects used in Experiment 2. On the left-hand side of the figure the jagged contours of interest are symmetric, on the right-hand side of the figure the contours of interest are repeated. In addition, each regularity condition is subdivided into facing and nonfacing versions of the contours of interest. Each row represents the presence and type of regularity of the curved irrelevant contours. 
contours of interest only, when both pairs of contours show the same regularity, this may lead to some facilitation effect because both (if detected) would yield the same response. In cases when irrelevant contours and contours of interest constitute regularities of a different type, this may cause some extra delay or errors in preparing the correct response because attention needs to be focused on the predefined target regularity between the predefined target contours. As before, these additional effects may play a stronger role in cases of symmetric contours of interest (e.g., possible interference from repeated irrelevant contours).

\section{Methods}

\section{Participants}

Twenty-six undergraduate students from the University of Leuven (18-24 years) performed the experiment and were given course credit. All participants reported normal or corrected-to-normal vision and were naïve regarding the goals and details of the experiment.

\section{Stimuli}

Eight new contours were created in 3DToolkit 2.5 (Electric Image, Inc.). Four jagged contours ( $\mathrm{Q}$ through $\mathrm{T}$ ) were created to be used as contours of interest, as well as four curved contours (W through $\mathrm{Z}$ ) to be used as irrelevant contours. In Figure 5, these two sets of four contours are shown. In this experiment, only two-objects situations were used. In pairing contours of interest with irrelevant contours to create single objects and in pairing two objects to define a trial, we avoided combinations with similar contours. So, because the contours of interest $\mathrm{Q}$ and $\mathrm{T}$, as well as $\mathrm{R}$ and $\mathrm{S}$, are fairly similar to each other, these contours were never presented in the same trial. The same holds for the relative similarity between the irrelevant contours $\mathrm{W}$ and $\mathrm{Z}$, as well as $\mathrm{X}$ and Y. To make the objects, each contour of interest was paired with two different irrelevant contours. For example, contour Q was paired with contour $\mathrm{W}$ to create one single object and with contour $\mathrm{Y}$ to create another single object.

The contours of interest and the irrelevant contours were also mirrored in a similar way as the contours of Experiment 1 . In addition, the contours of interest could be on the left side of the object or on the right side of the object (and vice versa for the irrelevant contours). This resulted in 64 unique objects: Four contours of interest $\times 2$ irrelevant contours $\times 2$ by mirroring the contours of interest $\times 2$ by mirroring the irrelevant contours $\times 2$ positions of the contours of interest. Next, each object was rendered four times. That is, each object was first placed to the right side of an image and then rendered twice: Once at an angle of $45^{\circ}$ to the right (with respect to the frontal plane) and once at an angle of $45^{\circ}$ to the left (with respect to the frontal plane). This process was then repeated, but now the object was placed to the left side of an image. Two hundred fifty-six objects were thus rendered in such a way that two-objects situations could be created, which were similar to the two-objects situations used in Experiment 1 (see also Figure 6). Note that, as mentioned, the visual extent between the contours of interest was always the same, for both facing stimuli and nonfacing stimuli but, as a result, the gap sizes varied between these conditions.

By systematically pairing the objects, 1024 trials were created: Four contours of interest $(\mathrm{Q}$ through $\mathrm{T}) \times 2$ types of regularity of contours of interest (symmetry or repetition) $\times 2$ for presence or absence of regularity of contours of interest $\times 2$ by mirroring the contours of interest $\times 4$ types of regularity of irrelevant contours (symmetry, repetition, and none; the latter was constructed twice for balancing) $\times 2$ by mirroring the irrelevant contours $\times 2$ orientations of the objects $\left(45^{\circ}\right.$ to the left and $45^{\circ}$ to the right $) \times 2$ for facing or nonfacing of contours of interest.

\section{Procedure}

The procedure was identical to that of Experiment 1 with the following exceptions. Participants were seated about $74 \mathrm{~cm}$ from the monitor. Facing stimuli covered an area of $5.1 \times 6.5 \mathrm{~cm}$ on the screen (i.e., a visual angle of about $5^{\circ}$ horizontally by $7^{\circ}$ vertically). Nonfacing stimuli covered an area of $3.6 \times 5.5 \mathrm{~cm}$ on the screen (i.e., a visual angle of about $4^{\circ}$ horizontally by $6^{\circ}$ vertically). Participants were carefully instructed to pay attention to the contours of interest (marked in red on an instruction sheet) and to ignore the irrelevant contours. They received a small practice session within which the experimenter could correct them when they made a mistake; they were allowed to move on to the real experimental trials only when they did not make more than two errors (which never occurred). The experiment was divided into two blocks: One block in which the contours of interest were facing and one block in which the contours of interest were nonfacing. The order of the blocks (facing first or nonfacing first) was counterbalanced across participants. In addition, half of the participants received trials with symmetric contours of interest; the other half of the participants received trials with repeated contours of interest. As a result, we now had a between-participants variable, called "group". Each participant received only 512 trials.

\section{Results and Discussion}

There was an overall $4.9 \%$ error rate across participants for all trials (5.3\% misses, $4.4 \%$ false alarms). Trials that did not contain a regularity of the contours of interest were not further analyzed. There was no speed-accuracy trade-off. Preliminary analyses revealed no main or interaction effects with respect to the variable "orientation", therefore the data were pooled across this variable. In addition, the two levels without regularity of the irrelevant contours (see Procedure) were pooled. For correct trials in which regularity of the contours of interest was present, mean RT was analyzed using a mixed ANOVA with the between-participant variable group (2) and repeated measures on variables facing (2) and regularity of irrelevant contours (3).

There was no significant difference between the two groups, $F(1,24)=1.43, p=.244$, but the trend was clearly 


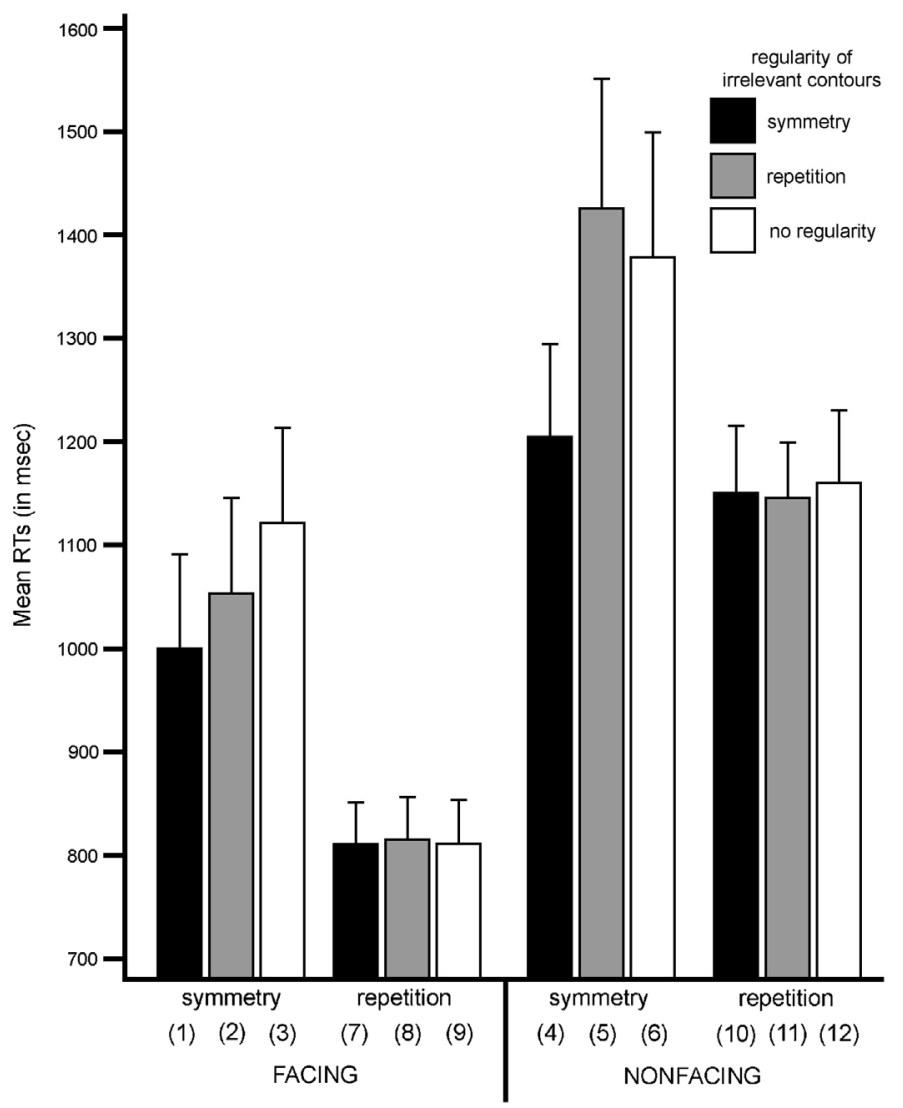

Figure 7. Results of Experiment 2. Graph of the mean reaction times (RTs) as a function of regularity of the contours of interest, facing and nonfacing versions of the contours of interest, and the regularity of the irrelevant contours. Error bars represent one standard error of the mean (representing the variability of each data point across participants). The numbers in parentheses on the $X$ axis refer to the panels in Figure 6.

in the direction of repetition being faster (Mean $=981 \mathrm{~ms}$, $S E M=126 \mathrm{~ms}$ ) than symmetry (Mean $=1194 \mathrm{~ms}, S E M=$ $126 \mathrm{~ms})$, rather than the other way around. A main effect was found for facing, $F(1,24)=45.54, p<.001, \eta_{\mathrm{P}}{ }^{2}=$ 0.655 . When the contours of interest were facing, participants responded faster (Mean $=935 \mathrm{~ms}, S E M=79 \mathrm{~ms}$ ) than when the contours of interest were nonfacing (Mean $=1240 \mathrm{~ms}$, $S E M=103 \mathrm{~ms}$ ). Second, a main effect of regularity of irrelevant contours was found, $F(2,23)=9.51, p<.005, \eta_{\mathrm{P}}^{2}=$ 0.453 . When the irrelevant contours were symmetric (Mean $=$ $1041 \mathrm{~ms}, S E M=80 \mathrm{~ms}$ ), responses were faster than when the irrelevant contours did not show a regularity (Mean $=1116 \mathrm{~ms}, S E M=95 \mathrm{~ms}$ ) or when the irrelevant contours were repeated (Mean $=1106 \mathrm{~ms}, S E M=91 \mathrm{~ms}$; the latter two means were not statistically different). The interaction effect between the regularity of irrelevant contours and group was also significant, $F(2,23)=10.02, p<.005, \eta_{\mathrm{P}}{ }^{2}=$ 0.466 . For the repetition-group, there were no significant differences between the levels of regularity of irrelevant contours. For the symmetry-group, responses were faster when the irrelevant contours were symmetric as compared to when the irrelevant contours did not show any regularity or when the irrelevant contours were repeated. Finally, the three-way inter- action of Facing $\times$ Regularity of irrelevant contours $\times$ Group was also significant, $F(2,23)=5.99, p<.01, \eta_{\mathrm{P}}{ }^{2}=0.343$. This means that the interaction between group and regularity of the irrelevant contours was different when the contours of interest were facing than when the contours of interest were nonfacing (see Figure 7). Other main and interaction effects were not significant. Note that the interaction effect between group and facing was not significant $(p=.535)$.

First, as can be seen on the left-hand side in Figure 7, when the contours of interest were facing, contrast comparisons revealed no significant differences between the levels of the regularity of the irrelevant contours for the repetition-group (all $F$-values $<1$ ). For the symmetry-group, average response time of the trials in which the irrelevant contours were symmetric was faster than when the irrelevant contours did not show any regularity, $F(1,24)=24.33, p<.001$, as well as when the irrelevant contours were repeated, $F(1,24)=7.96$, $p<.01$. In addition, for the symmetry-group, the latter two conditions also differed statistically, $F(1,24)=6.22$, $p<.05$. Second, as can be seen on the right-hand side in Figure 7, when the contours of interest were nonfacing, contrast comparisons revealed no significant differences between the levels of the regularity of the irrelevant contours 
for the repetition-group (all $p$-values $>.3$ ). For the symmetrygroup, average response time of the trials in which the irrelevant contours were symmetric was faster than when the irrelevant contours did not show a regularity, $F(1,24)=21.65, p<.001$, as well as when the irrelevant contours were repeated, $F(1,24)=35.98, p<.001$. Here, the latter two conditions did not differ statistically $(p>.1)$.

First of all, the current results show that symmetry is often, but not always, easier to detect than repetition. This corresponds to earlier findings with different paradigms stimuli. For example, using a sequential matching task, Koning and Van Lier (2006) found that 3-D object matching was easier for symmetric objects than for asymmetric objects only when the 3-D structures of the objects are visible, not when accidental viewpoints of objects are involved. Second, in contrast to what could be expected when the repetition advantage for two-objects situations was due to lock-and-key matching, there was no symmetry advantage when this matching strategy was made unlikely by switching the positions of the objects. On the contrary, repetition was still faster in many conditions, suggesting that the two-objects advantage for repetition over symmetry is due to structural differences and not due to matching strategies. However, although repetition was faster than symmetry in many conditions, the main effect of regularity was not statistically significant, partly because it could not be tested in the most powerful way in our design (because it was a between-subjects variable).

Moreover, consistent with the structural account, the type of regularity between the irrelevant contours affected the symmetry judgments but not the repetition judgments. In contrast to what could have been expandected when participants used a matching strategy for repetitions, the results showed that repetition detection was not affected by the different types of irrelevant contours, irrespective of whether the contours of interest were facing or nonfacing. For the symmetry-group, the type of irrelevant contour did result in reliable performance differences: In both cases (facing and nonfacing contours), symmetry between contours of interest was the least difficult to detect when it was accompanied by symmetry between irrelevant contours. That is, when the irrelevant contours were symmetric, performance was always better than when the irrelevant contours were repeated or when the irrelevant contours did not show any regularity. It thus appears that in case of symmetry detection in the two-objects situations used here, not only local symmetry detection of the contours of interest, but also the global regularity of a display, which includes the irrelevant contours, influenced participants' performance. This modulatory effect was completely absent in the conditions where repetition had to be detected.

\section{General Discussion}

The present study started from a simple but intriguing observation: Symmetry is generally easier to detect than repetition, except when the repetition concerns a pair of contours belonging to two different objects (assuming the original terminology introduced in earlier studies; but see
Csatho et al., 2003, for a more formally correct treatment). We wanted to find out whether this finding (Baylis \& Driver, 1995; Bertamini et al., 1997) reflects a fundamental characteristic of object coding or reflects a specific positioning of the regular contours (allowing, for example, a matching strategy). A possible way to start to explain this result would be to refer to the fact that symmetry is an intrinsic property of objects (e.g., animal bodies, human faces, many artifacts) whereas repetition has more to do with specific relations between-objects (e.g., a broken plate or the pieces of a puzzle). In light of the relative frequency and importance of symmetry and repetition within and between-objects, even though we do not have any data about the actual occurrence of this in the natural environment, it would not be unlikely if the visual system's detection of regularities would show a within-object benefit for symmetry and a between-objects benefit for repetition. In our experiments, the participants' task was to determine whether a pair of target contours showed symmetry or not, or repetition or not (in different blocks in Experiment 1 and in different groups of participants in Experiment 2). The stimuli consisted of protruded 3-D objects rotated in depth, so that the 2-D contours did not match exactly but required an object interpretation to determine their regularity.

The interaction between number of objects and type of regularity in Experiment 1 was quite strong. Symmetry was detected better when the target contours belonged to one object rather than to two objects. Alternatively, for repetition, the opposite was found, that is, faster RTs when contours belonging to two objects had to be judged as compared to one object. Object structure must have played a major role in this pattern of results because 2-D contour regularity was reduced by using protruded 3-D objects turned away from the frontoparallel plane. We suspect that this specific interaction effect was even stronger in our experiment with 3-D objects than in the previous studies with 2-D images. Unfortunately, Baylis and Driver (1995) did not test the two types of regularity in a single experiment. They do report small but statistically significant differences in RT between the one-object and two-objects situations for both symmetry and repetition. Bertamini et al. (1997) did investigate both regularities simultaneously in several experiments but they did not always obtain a significant interaction. The previous results in the literature thus seem quite a bit weaker compared to the relatively large differences in RT that were found here.

However, a direct comparison of the current results with those of previous studies by Baylis and Driver (1995) or Bertamini et al. (1997) is complicated by several methodological differences (e.g., stimuli). Therefore, we performed a small control experiment to be able to compare the effects for 3-D objects with those for 2-D images more directly. Half of the 3-D objects from Experiment 1 were rendered from a fully frontal viewpoint such that only flat (2-D) surfaces were now visible. In a repeated-measures ANOVA, we found a three-way interaction (Regularity $\times$ Number of objects $\times$ Experiment) that was highly significant, $F(1,38)=9.58, p<.005, \eta_{\mathrm{P}}^{2}=0.2$. That is to say, whereas for the 2-D objects, the regularity by number of objects interaction was not significant $(F=3.02, p>.05)$ for the 
3-D objects, the same interaction was highly significant $(F=13.14, p<.005)$. Of course, as shown in the literature, with more power (e.g., more trials) the same interaction can be significant for 2-D images as well (e.g., Baylis \& Driver, 1995; Bertamini et al., 1997). Our results from a direct comparison, however, indicate that even with a small number of (comparable) trials, the regularity by number of objects interaction is already strongly significant using 3-D objects, while it is not significant with 2-D images.

To be able to attribute these findings of Experiment 1 even less ambiguously to the visual system's intrinsic coding of object regularities, an alternative explanation had to be ruled out. Perhaps the two-objects benefit for repetition resulted from a special matching strategy between repeated contours of adjacent objects (cf., Bertamini et al.'s lockand-key or Baylis \& Driver's jigsaw matching, but now in depth). Such a strategy was made unlikely by manipulating the position of the two objects in the displays of Experiment 2. In addition, the strength of the perceptual grouping of the two objects varied as a function of the width of the gap and the relation between the nontarget contours. Overall, there was a strong effect of the position of the two objects. Faster responses were made when the target contours were facing one another than when they were nonfacing. However, it was not the case that a symmetry advantage was obtained when the object positions were switched to prevent the direct matching of repeated contours. Repetition of target contours was detected faster in many conditions and this was not influenced by the additional regularity between the nontarget contours (although they were separated by a smaller gap). In contrast, symmetry detection between target contours was affected by the additional regularity between the nontarget contours and it became only as fast as repetition detection when the displays showed overall symmetry.

Recently, Bertamini, Friedenberg, and Argyle (2002) suggested that what matters in case of repetition detection is comparison across a gap versus comparison across a surface. More specifically, they suggested that the repetition advantage with two objects is better described as a gap advantage rather than by lock-and-key matching as Bertamini et al. (1997) previously did. Using closure to define figures separated from a ground, what Bertamini et al. (2002) found was that repetition detection across a gap was easier than across a surface, irrespective of the number of interpreted objects. Additionally, Bertamini et al. (2002) did not find any performance difference in the repetition detection task between-participants who reported using a matching strategy and participants who did not report such a strategy. The conclusion by Bertamini et al. (2002) that matching strategies cannot explain the two-objects advantage for repetition (based on subjective reports) is in line with our finding in Experiment 2 that the symmetry advantage over repetition did not return when the spatial positioning of the objects was reversed. However, in contrast to Bertamini et al. (2002) we argue that it is the number of objects rather than a gap advantage that is responsible for the two-objects advantage for repetition over symmetry. That is, using only closure of contours to establish comparisons across a gap versus comparisons across a surface, as
Bertamini et al. (2002) did, has probably led to ambiguous figure-ground segmentation. In case of our 3-D stimuli, this ambiguity has been ruled out and our results suggest an inherent two-objects advantage for repetition and a oneobject advantage for symmetry.

Note that by stereoscopically presenting stimulus displays comparable to those of Bertamini et al. (1997) (see Figure 1), it is possible to compare the contributions of the different factors more directly to one another: Bertamini et al.'s (2002) gap advantage, Bertamini et al.'s (1997) lockand-key matching strategy, and a structural description account (as suggested here). That is, using a two-monitor setup and laterally displaced figure elements allows stereofusion to create a compelling percept of different depth layers in which regularities can then be presented that have to be compared across a gap or across a surface. This specific issue of comparison across a surface versus a gap will be the topic of a future study (Koning, Kogo, \& Wagemans, 2008 ), because it goes beyond our initial intention of contrasting structural differences with matching strategies.

In addition to the role of figure-ground segmentation and object structure, Experiments 1 and 2 also required participants to judge object-based regularities rather than pure image-based regularities. The fact that people could do this rather well, producing only a small number of errors, is in agreement with previous studies on the detection and use of nonorthogonal regularities in dot patterns and polygons (Van der Vloed, Csatho, \& Van der Helm, 2005; Wagemans, 1992, 1993), blob patterns (Van der Vloed et al., 2005), surfaces (Saunders \& Knill, 2001), cube-like figures (Van Lier \& Wagemans, 1999), and cross-like figures (Willems \& Wagemans, 2000). In addition, our use of nonorthogonal regularities strengthens the case for the role of object structure in explaining the interaction between type of regularity and number of objects. This dominance for object-based information is in line with previous results on, for example, object-based connectedness between-objects (Koning \& Van Lier, 2003, 2005), compared to cases where image-based and object-based connectedness cannot be distinguished (Van Lier \& Wagemans, 1998). In the case of objects such as the ones used by Baylis and Driver (1995) and Bertamini et al. (1997), there is a congruency between what is presented (a regular image) and what is represented (a regular object), whereas in the case of the objects used here the regularities are only available on the basis of their interpretations (from an imperfect regularity in the image to a perfectly regular object). The fact that the preserved image-based regularity of those previous studies has not strengthened the effects (rather the contrary) suggests that the visual system does not take advantage of any extra image-based qualities that may be present, a conclusion that is in line with previous suggestions (Koning \& Van Lier, 2005; Saiki \& Hummel, 1998).

Overall, the current experiments provide strong evidence that intrinsic coding of regularities is responsible for the interaction between type of regularity and number of objects, not matching strategies. More importantly, however, the 3-D objects used here resulted in stronger effects as compared to the 2-D objects used previously. As mentioned before, in case of 2-D images segmenting figures from 
ground is not all that straightforward. It is well-known that figure-ground organization is one of the major tasks that the visual system has to solve to make sense of the visual input. Some regions belong to figures and others to the background continuing behind them, and the visual system must try to establish the correct figure-ground assignment on the basis of the available information. However, regularities themselves have been considered to be processed relatively early (perhaps even preattentively) and also to influence segmentation (Bahnsen, 1928; Kanizsa \& Gerbino, 1976; Van der Helm \& Leeuwenberg, 1996). Thus, by making the intended figure-ground segmentation less ambiguous (by using 3-D instead of 2-D objects) we were able to more directly investigate the role of intrinsic coding of the regularities. In Experiment 1, the protruded and slanted 3-D objects made it difficult for observers to switch figure-ground interpretation. This enhanced the objectness of the gray figures and reduced the figural qualities of the white region. As a result, we found a strong interaction between number of objects and type of regularity.

In total, although visual regularities can be constructed formally in several ways and the specific way of connecting pairs of contours (i.e., the context) also influences formal regularity descriptions, the present experiments indicate that the interaction between type of regularity and number of objects strongly depends on object structure, not on specific strategies participants might use. Even though we might encounter visual regularities like symmetry more often within a single object, and repetition more often between multiple objects, making a matching strategy not unlikely, we have shown that the symmetry advantage for one-object displays and the repetition advantage for two-objects displays reflect an intrinsic property of the way in which the visual system encodes visual regularities between contours within and across objects.

\section{Acknowledgments}

Arno Koning was supported by a fellowship from the Research Council from the University of Leuven ( $F / 04 /$ 075). The authors would also like to thank Marco Bertamini, Peter Van der Helm, Naoki Kogo, and Tessa de Wit for their helpful comments and suggestions on a previous version of this manuscript. Parts of this study were presented at the 29th European Conference on Visual Perception (ECVP) in St. Petersburg, Russia.

\section{References}

Bahnsen, P. (1928). Eine untersuchung über Symmetrie und Asymmetrie bei visuellen Wahrnehmungen. Zeitschrift für Psychologie, 108, 355-361.

Baylis, G. C., \& Driver, J. (1994). Parallel computation of symmetry but not repetition within single visual shapes. Visual Cognition, 1, 377-400.

Baylis, G. C., \& Driver, J. (1995). Obligatory edge assignment in vision: The role of figure and part segmentation in symmetry detection. Journal of Experimental Psychology: Human Perception and Performance, 21, 1323-1342.
Baylis, G. C., \& Driver, J. (2001). Perception of symmetry and repetition within and across visual shapes: Part-based descriptions and object-based attention. Visual Cognition, 8, 163-196.

Bertamini, M., Friedenberg, J., \& Argyle, L. (2002). No withinobject advantage for detection of rotation. Acta Psychologica, 111, 59-81.

Bertamini, M., Friedenberg, J., \& Kubovy, M. (1997). Detection of symmetry and perceptual organization: The way a lock-and-key process works. Acta Psychologica, 95, 119-140.

Corballis, M. C., \& Roldan, C. E. (1974). On the perception of symmetrical and repeated patterns. Perception \& Psychophysics, 16, 136-142.

Csatho, A., Van der Vloed, G., \& Van der Helm, P. A. (2003). Blobs strengthen repetition but weaken symmetry. Vision Research, 43, 993-1007.

Hoffman, D. D., \& Richards, W. A. (1984). Parts of recognition. Cognition, 18, 65-96.

Jenkins, B. (1983). Component processes in the perception of bilaterally symmetric dot textures. Perception \& Psychophysics, 34, 433-440.

Kanizsa, G., \& Gerbino, W. (1976). Convexity and symmetry in figure-ground organization. In M. Henle (Ed.), Art and artifact (pp. 25-32). New York: Springer.

Koning, A., Kogo, N., \& Wagemans, J. (2008). Detection of symmetry and repetition within objects and across holes. Manuscript in preparation.

Koning, A., \& Van Lier, R. (2003). Object-based connectedness facilitates matching. Perception \& Psychophysics, 65, 1094 1102.

Koning, A., \& Van Lier, R. (2005). From interpretation to segmentation. Psychonomic Bulletin \& Review, 12, 917-924.

Koning, A., \& Van Lier, R. (2006). No symmetry advantage when object matching involves accidental viewpoints. Psychological Research, 70, 52-58.

Kubovy, M., \& Wagemans, J. (1995). Grouping by proximity and multistability in dot lattices: A quantitative Gestalt theory. Psychological Science, 6, 225-234.

Kubovy, M., Holcombe, A. O., \& Wagemans, J. (1998). On the lawfulness of grouping by proximity. Cognitive Psychology, 35, 71-98

Mach, E. (1959). The analysis of sensations. New York: Dover (Original work published in 1885).

Palmer, S. E. (1983). The psychology of perceptual organization: A transformational approach. In J. Beck, B. Hope, \& A. Rosenfeld (Eds.), Human and machine vision (pp. 269-339). New York: Academic Press.

Rubin, E. (1921). Visuell wahrgenommene Figuren [Visually perceived figures]. Copenhagen, Denmark: Gyldendal.

Saiki, J., \& Hummel, J. E. (1998). Connectedness and the integration of parts within relations in shape perception. Journal of Experimental Psychology: Human Perception and Performance, 24, 227-251.

Saunders, J. A., \& Knill, D. C. (2001). Perception of 3D surface orientation from skew symmetry. Vision Research, 41, 31633183.

Strother, L., \& Kubovy, M. (2003). Perceived complexity and the grouping effect in band patterns. Acta Psychologica, 114, 229-244.

Tyler, C. W. (1995). Empirical aspects of symmetry perception. Spatial Vision, 9, 1-7.

Van der Helm, P. A., \& Leeuwenberg, E. L. J. (1991). Accessibility, a criterion for regularity and hierarchy in visual pattern codes. Journal of Mathematical Psychology, $35,151-213$

Van der Helm, P. A., \& Leeuwenberg, E. L. J. (1996). Goodness of visual regularities: A nontransformational approach. Psychological Review, 103, 429-456. 
Van der Helm, P. A., \& Leeuwenberg, E. L. J. (1999). A better approach to goodness: Reply to Wagemans (1999). Psychological Review, 106, 622-630.

Van der Vloed, G., Csatho, A., \& Van der Helm, P. A. (2005). Symmetry and repetition in perspective. Acta Psychologica, 120, 74-92.

Van Lier, R., \& Wagemans, J. (1998). Effects of physical connectivity on the representational unity of multi-part configurations. Cognition, 69, B1-B9.

Van Lier, R., \& Wagemans, J. (1999). From images to objects: Global and local completions of self-occluded parts. Journal of Experimental Psychology: Human Perception and Performance, 25, 1721-1741.

Wagemans, J. (1992). Perceptual use of nonaccidental properties. Canadian Journal of Psychology, 46, 236-279.

Wagemans, J. (1993). Skewed symmetry: A nonaccidental property used to perceive visual forms. Journal of Experimental Psychology: Human Perception and Performance, 19, 364-380.

Wagemans, J. (1995). Detection of visual symmetries. Spatial Vision, 9, 9-32.

Wagemans, J. (1997). Characteristics and models of human symmetry detection. Trends in Cognitive Sciences, 1, 346352.

Wagemans, J. (1999). Toward a better approach to goodness: Comments on Van der Helm and Leeuwenberg (1996). Psychological Review, 106, 610-621.
Wagemans, J., Van Gool, L., \& d'Ydewalle, G. (1991). Detection of symmetry in tachistoscopically presented dot patterns: Effects of multiple axes and skewing. Perception \& Psychophysics, 50, 413-427.

Wagemans, J., Van Gool, L., Swinnen, V., \& Van Horebeek, J. (1993). Higher-order structure in regularity detection. Vision Research, 33, 1067-1088.

Willems, B., \& Wagemans, J. (2000). The viewpoint-dependency of veridicality: Psychophysics and modeling. Vision Research, 40, 3017-3027.

Received March 5, 2007

Revision received September 20, 2007

Accepted October 8, 2007

Arno Koning

Nijmegen Institute for Cognition and Information

Radboud University

P.O. Box 9104

6500 HE Nijmegen

The Netherlands

Tel. +31 (0) 243612635

Fax $+31(0) 243616066$

E-mail arno.koning@nici.ru.nl 